\title{
TWO-VARIABLE WIMAN-VALIRON THEORY AND PDES
}

\author{
P. C. Fenton and John Rossi \\ University of Otago, Department of Mathematics and Statistics \\ P.O. Box 56, Dunedin, New Zealand; pfenton@maths.otago.ac.nz \\ Virginia Tech, Department of Mathematics \\ Blacksburg, VA 24061-0123, U.S.A.; rossij@math.vt.edu
}

\begin{abstract}
We show how Wiman-Valiron techniques can be applied to partial differential equations in two complex variables.
\end{abstract}

\section{Introduction}

Wiman-Valiron theory involves the analysis of entire functions by means of the maximum term and central index. For a function of two variables,

$$
f\left(z_{1}, z_{2}\right)=\sum_{n_{1}=0}^{\infty} \sum_{n_{2}=0}^{\infty} a_{n_{1}, n_{2}} z_{1}^{n_{1}} z_{2}^{n_{2}},
$$

the maximum term is

$$
\mu\left(r_{1}, r_{2}\right)=\max \left\{\left|a_{n_{1}, n_{2}}\right| r_{1}^{n_{1}} r_{2}^{n_{2}}: m, n=0,1,2, \ldots\right\}, \quad r_{1}, r_{2} \geq 0,
$$

and, if $N_{1}=N_{1}\left(r_{1}, r_{2}\right)$ and $N_{2}=N_{2}\left(r_{1}, r_{2}\right)$ are non-negative integers such that

$$
\mu\left(r_{1}, r_{2}\right)=\left|a_{N_{1}, N_{2}}\right| r_{1}^{N_{1}} r_{2}^{N_{2}},
$$

the central index is $\mathbf{N}=\mathbf{N}\left(r_{1}, r_{2}\right)=\left(N_{1}, N_{2}\right)$. The central index is not well-defined (that is, is not unique) for certain values of $\left(r_{1}, r_{2}\right)$; for most purposes the central index for those values may be taken to be any $\mathbf{N}$ for which (3) holds.

In $[1,2]$ the first author developed Wiman-Valiron techniques for entire functions of two variables. The main theorem of [2] concerns the behaviour of the partial derivatives at points $\left(z_{1}, z_{2}\right)$ for which $\left(\left|z_{1}\right|,\left|z_{2}\right|\right)$ lies in the so-called normal set. We use the notation

$$
f^{p_{1}, p_{2}}=\frac{\partial^{p_{1}+p_{2}} f}{\partial z_{1}^{p_{1}} \partial z_{2}^{p_{2}}}
$$

The main theorem of [2] concerns the behaviour of the partial derivatives of $f$ at points $\left(z_{1}, z_{2}\right)$ for which $\left(\left|z_{1}\right|,\left|z_{2}\right|\right)$ lies in the so-called normal set, that is the set of points $\left(r_{1}, r_{2}\right), r_{1}, r_{2} \geq 0$, for which $\left|a_{n_{1}, n_{2}}\right| r_{1}^{n_{1}} r_{2}^{n_{2}} / \mu\left(r_{1}, r_{2}\right)$ is suitably bounded for all $n_{1}, n_{2}$; see [1, pp. 4406-7] for details.

Theorem A. [2, Theorem 3] Suppose that $\left(r_{1}, r_{2}\right)$ is normal and that $z_{1}$ and $z_{2}$ are such that $\left|z_{1}\right|=r_{1},\left|z_{2}\right|=r_{2}$ and $\left|f\left(z_{1}, z_{2}\right)\right|=M\left(r_{1}, r_{2}\right)$, where

$$
M\left(r_{1}, r_{2}\right)=\max _{\left|\zeta_{1}\right|=r_{1},\left|\zeta_{2}\right|=r_{2}}\left|f\left(\zeta_{1}, \zeta_{2}\right)\right| \text {. }
$$

doi:10.5186/aasfm.2010.3535

2000 Mathematics Subject Classification: Primary 32A15.

Key words: Wiman-Valiron theory, entire function of two variables. 
Let

$$
N^{*}=\max \left\{N_{1}, N_{2}\right\}, \quad N_{*}=\min \left\{N_{1}, N_{2}\right\} .
$$

For any non-negative integers $p_{1}$ and $p_{2}$, there are constants $C=C\left(p_{1}, p_{2}\right)$ and $N_{0}=N_{0}\left(p_{1}, p_{2}\right)$ such that, if $N_{*} \geq N_{0}$ and

$$
\log N^{*} \leq \frac{N_{*}}{240\left(p_{1}+p_{2}+1\right)\left(\log N_{*}\right)^{2}},
$$

then

$$
f^{p_{1}, p_{2}}\left(z_{1}, z_{2}\right)=(1+\sigma)\left(\frac{N_{1}}{z_{1}}\right)^{p_{1}}\left(\frac{N_{2}}{z_{2}}\right)^{p_{2}} f\left(z_{1}, z_{2}\right),
$$

where

$$
|\sigma| \leq C \sqrt{\frac{\log N^{*}\left(\log N_{*}\right)^{2}}{N_{*}}} .
$$

An example [1, p. 228] shows that $f^{p_{1}, p_{2}}\left(z_{1}, z_{2}\right)$ and $\left(N_{1} / z_{1}\right)^{p_{1}}\left(N_{2} / z_{2}\right)^{p_{2}} f\left(z_{1}, z_{2}\right)$ may bear no significant relationship if (5) just fails. For

$$
f\left(z_{1}, z_{2}\right)=\sum_{n=0}^{\infty} \frac{z_{1}^{n}+z_{1}^{n-1} z_{2}+\cdots+z_{1} z_{2}^{n-1}+z_{2}^{n}}{n !},
$$

the central index is $\left(N_{1}, 0\right)$ if $\left|z_{1}\right|>\left|z_{2}\right|,\left(0, N_{2}\right)$ if $\left|z_{1}\right|<\left|z_{2}\right|$ and is not well-defined if $\left|z_{1}\right|=\left|z_{2}\right|$. Thus (6) may fail for all mixed partial derivatives, and in fact (6) may hold only for $z_{1}$ partial derivatives, or only for $z_{2}$ partial derivatives.

We call the complement of the normal set in the first quadrant the exceptional set, and denote it by $E$. Estimates of the exceptional set are given in [1] in terms of two-dimensional logarithmic measure, $r_{1}^{-1} r_{2}^{-1} d r_{1} d r_{2}$. It is shown that, for any entire function,

$$
\iint_{E \cap([1, R \times[1, R])} \frac{d r_{1} d r_{2}}{r_{1} r_{2}}<3 \log R,
$$

for all $R \geq 1$. But other estimates are possible using the argument of [1]; for example, for any $R_{1} \geq 1$ and $R_{2} \geq 1$,

$$
\iint_{E \cap\left(\left[R_{1}, R_{1}^{2}\right] \times\left[R_{2}, R_{2}^{2}\right]\right)} \frac{d r_{1} d r_{2}}{r_{1} r_{2}}<3\left(\log R_{1}+\log R_{2}\right) .
$$

Lemma 2 below gives another estimate for the exceptional set.

The analogue of Theorem $\mathrm{A}$ in one dimension has had important applications in determining the existence and estimating the growth of entire solutions of ordinary differential equations with entire coefficients; see [4, Chapter 4]. For example, entire solutions of

$$
f^{\prime \prime}(z)+P(z) f(z)=0,
$$

where $P$ is a polynomial of degree $n$, satisfy

$$
\frac{N(r)^{2}}{r^{2}}=(c+o(1)) r^{n}
$$


where $c>0$ is a constant, whence $N(r)$ grows like $r^{(n+2) / 2}$. Since $N$ is comparable to $\log M$ on the normal set, and since the exceptional set is relatively small, this gives us that the order of every solution to $(9)$ is $(n+2) / 2$.

In light of Theorem A, it is natural to ask whether we can make similar growth estimates for entire solutions to linear partial differential equations in two complex variables when the coefficients are polynomials. One (insurmountable) obstacle is that, since we can prescribe an initial function of arbitrary growth, the best we can hope for is a lower bound on the growth of solutions. For example, the general solution to $f^{1,0}\left(z_{1}, z_{2}\right)=f\left(z_{1}, z_{2}\right)$ is $f\left(z_{1}, z_{2}\right)=\Phi\left(z_{2}\right) e^{z_{1}}$, where $f\left(0, z_{2}\right)=\Phi\left(z_{2}\right)$ may be any entire function.

A second obstacle is that, unlike in the one dimensional case, our solution $f$ must satisfy (5), which is a priori impossible to check. To get around this, we define in the next section an associated function $\mathscr{F}$ which depends on $f$ and satisfes (5). Given a partial differential equation in $f$, we find the associated equation in $\mathscr{F}$ and use (6). A lower bound on the growth of $\mathscr{F}$ (and hence $f$ ) then follows as in the one dimensional case. This method is the main content of the paper. We will, however, also prove a version of Theorem A that does not require the hypothesis (5).

\section{An associated function}

We define

$$
\mathscr{F}\left(\zeta_{1}, \zeta_{2}\right)=f\left(\zeta_{1}^{2} \zeta_{2}, \zeta_{1} \zeta_{2}^{2}\right)=\sum_{n_{1}=0}^{\infty} \sum_{n_{2}=0}^{\infty} a_{n_{1}, n_{2}} \zeta_{1}^{2 n_{1}+n_{2}} \zeta_{2}^{2 n_{2}+n_{1}}
$$

For $\mathscr{F}$, the inequality (5) is trivially satisfied whenever its central index, $\mathscr{N}=$ $\left(\mathscr{N}_{1}, \mathscr{N}_{2}\right)$ say, is large, and therefore

$$
\mathscr{F}^{p_{1}, p_{2}}\left(\zeta_{1}, \zeta_{2}\right)=(1+\sigma)\left(\frac{\mathscr{N}_{1}}{\zeta_{1}}\right)^{p_{1}}\left(\frac{\mathscr{N}_{2}}{\zeta_{2}}\right)^{p_{2}} \mathscr{F}\left(\zeta_{1}, \zeta_{2}\right),
$$

for all $\left(\zeta_{1}, \zeta_{2}\right)$ such that $\left(\left|\zeta_{1}\right|,\left|\zeta_{2}\right|\right)$ is normal for $\mathscr{F},\left|\mathscr{F}\left(\zeta_{1}, \zeta_{2}\right)\right|=\mathscr{M}\left(\left|\zeta_{1}\right|,\left|\zeta_{2}\right|\right)$ and $\mathscr{N}\left(\left|\zeta_{1}\right|,\left|\zeta_{2}\right|\right)$ is large.

The central indices of $f$ and $\mathscr{F}$ are connected by the equations:

$$
\begin{aligned}
& 3 N_{1}\left(r_{1}^{2} r_{2}, r_{1} r_{2}^{2}\right)=2 \mathscr{N}_{1}\left(r_{1}, r_{2}\right)-\mathscr{N}_{2}\left(r_{1}, r_{2}\right), \\
& 3 N_{2}\left(r_{1}^{2} r_{2}, r_{1} r_{2}^{2}\right)=2 \mathscr{N}_{2}\left(r_{1}, r_{2}\right)-\mathscr{N}_{1}\left(r_{1}, r_{2}\right) .
\end{aligned}
$$

For, if $N_{1}$ and $N_{2}$ are defined by $\mathscr{N}_{1}=2 N_{1}+N_{2}$ and $\mathscr{N}_{2}=N_{1}+2 N_{2}$, then for all $j$ and $k$,

$$
\left|a_{N_{1}, N_{2}}\right| r_{1}^{\mathscr{N}_{1}} r_{2}^{\mathcal{N}_{2}} \geq\left|a_{j, k}\right| r_{1}^{2 j+k} r_{2}^{j+2 k}
$$

that is

$$
\left|a_{N_{1}, N_{2}}\right|\left(r_{1}^{2} r_{2}\right)^{N_{1}}\left(r_{1} r_{2}^{2}\right)^{N_{2}} \geq\left|a_{j, k}\right|\left(r_{1}^{2} r_{2}\right)^{j}\left(r_{1} r_{2}^{2}\right)^{k},
$$

and thus $\left(N_{1}, N_{2}\right)$ is the central index of $f$ at $\left(r_{1}^{2} r_{2}, r_{1} r_{2}^{2}\right)$. (The same calculation shows that $\left(r_{1}, r_{2}\right)$ is normal for $\mathscr{F}$ if and only if $\left(r_{1}^{2} r_{2}, r_{1} r_{2}^{2}\right)$ is normal for $f$; see [1, p. 4407].)

We will prove the following theorem. 
Theorem 1. Suppose that $\zeta_{1}$ and $\zeta_{2}$ are such that $\left(\left|\zeta_{1}\right|,\left|\zeta_{2}\right|\right)$ is normal for $\mathscr{F}$ and $\left|\mathscr{F}\left(\zeta_{1}, \zeta_{2}\right)\right|=\mathscr{M}\left(\left|\zeta_{1}\right|,\left|\zeta_{2}\right|\right)$. Write $z_{1}=\zeta_{1}^{2} \zeta_{2}$ and $z_{2}=\zeta_{1} \zeta_{2}^{2}$. For all $p_{1} \geq 0$ and $p_{2} \geq 0$,

$$
f^{p_{1}, p_{2}}\left(z_{1}, z_{2}\right)=(1+\sigma)\left(\frac{N_{1}}{z_{1}}\right)^{p_{1}}\left(\frac{N_{2}}{z_{2}}\right)^{p_{2}} f\left(z_{1}, z_{2}\right)
$$

where

$$
|\sigma|=O\left(\frac{N^{* p_{1}+p_{2}-1 / 2}\left(\log N^{*}\right)^{3 / 2}}{N_{1}^{p_{1}} N_{2}^{p_{2}}}\right) .
$$

Notice that $\sigma$ may be large, and (13) effectively useless, if $N_{1}$ and $N_{2}$ are significantly different. In view of Theorem $\mathrm{A}$ and the remarks following it, this is to be expected.

To prove Theorem 1 , we first note that, for all $p_{1} \geq 0$ and $p_{2} \geq 0$,

$$
z_{1}^{p_{1}} z_{2}^{p_{2}} f^{p_{1}, p_{2}}\left(z_{1}, z_{2}\right)=\sum_{k_{1}+k_{2}=0}^{p_{1}+p_{2}} \alpha\left(k_{1}, k_{2}, p_{1}, p_{2}\right) \zeta_{1}^{k_{1}} \zeta_{2}^{k_{2}} \mathscr{F}^{k_{1}, k_{2}}\left(\zeta_{1}, \zeta_{2}\right)
$$

where $\alpha\left(k_{1}, k_{2}, p_{1}, p_{2}\right)$ is a real constant for each $k_{1}, k_{2}, p_{1}, p_{2}$.

Certainly (15) is true if $p_{1}=0$ and $p_{2}=0$, and differentiating (15) partially with respect to $\zeta_{1}$ and using

$$
\frac{\partial \zeta_{1}}{\partial z_{1}}=\frac{2 \zeta_{1}}{3 z_{1}}, \quad \frac{\partial \zeta_{2}}{\partial z_{1}}=-\frac{\zeta_{2}}{3 z_{1}}
$$

we obtain

$$
\begin{aligned}
& p_{1} z_{1}^{p_{1}} z_{2}^{p_{2}} f^{p_{1}, p_{2}}\left(z_{1}, z_{2}\right)+z_{1}^{p_{1}+1} z_{2}^{p_{2}} f^{p_{1}+1, p_{2}}\left(z_{1}, z_{2}\right) \\
& =\sum_{k_{1}+k_{2}=0}^{p_{1}+p_{2}} \alpha\left(k_{1}, k_{2}, p_{1}, p_{2}\right)\left(\frac{2}{3} k_{1} \zeta_{1}^{k_{1}} \zeta_{2}^{k_{2}}-\frac{1}{3} k_{2} \zeta_{1}^{k_{1}} \zeta_{2}^{k_{2}}\right) \mathscr{F}^{k_{1}, k_{2}}\left(\zeta_{1}, \zeta_{2}\right) \\
& \quad+\sum_{k_{1}+k_{2}=0}^{p_{1}+p_{2}} \alpha\left(k_{1}, k_{2}, p_{1}, p_{2}\right)\left(\frac{2}{3} \zeta_{1}^{k_{1}+1} \zeta_{2}^{k_{2}} \mathscr{F}^{k_{1}+1, k_{2}}\left(\zeta_{1}, \zeta_{2}\right)-\frac{1}{3} \zeta_{1}^{k_{1}} \zeta_{2}^{k_{2}+1} \mathscr{F}^{k_{1}, k_{2}+1}\left(\zeta_{1}, \zeta_{2}\right)\right),
\end{aligned}
$$

and (15) follows with $p_{1}$ replaced by $p_{1}+1$. A similar result is obtained on differentiating (15) partially with respect to $z_{2}$, and (15) follows by induction.

Next we show that the terms on the right hand side of (15) that involve derivatives of $\mathscr{F}$ of highest order - that is, derivatives of order $p_{1}+p_{2}$-are

$$
\sum_{i=0}^{p_{1}} \sum_{j=0}^{p_{2}} B\left(i, j, p_{1}, p_{2}\right) \zeta_{1}^{i+j} \zeta_{2}^{p_{1}+p_{2}-i-j} \mathscr{F}^{i+j, p_{1}+p_{2}-i-j}\left(\zeta_{1}, \zeta_{2}\right)
$$

where

$$
B\left(i, j, p_{1}, p_{2}\right)=C_{i}^{p_{1}} C_{j}^{p_{2}}\left(-\frac{1}{3}\right)^{p_{1}-i+j}\left(\frac{2}{3}\right)^{p_{2}+i-j}
$$

and $C_{i}^{p}$ is the usual binomial coefficient. This is clearly true if $p_{1}=0$ and $p_{2}=0$. Also, if the terms of highest order are given by (16) and (17) for certain values of 
$p_{1}$ and $p_{2}$ then, differentiating (15) partially with respect to $z_{1}$, the terms involving derivatives of highest order for the values $p_{1}+1$ and $p_{2}$ are

$$
C_{i}^{p_{1}} C_{j}^{p_{2}}\left(-\frac{1}{3}\right)^{p_{1}-i+j}\left(\frac{2}{3}\right)^{p_{2}+i-j+1} \zeta_{1}^{i+j+1} \zeta_{2}^{p_{1}+p_{2}-i-j} \mathscr{F}^{i+j+1, p_{1}+p_{2}-i-j}\left(\zeta_{1}, \zeta_{2}\right)
$$

and

$$
C_{i}^{p_{1}} C_{j}^{p_{2}}\left(-\frac{1}{3}\right)^{p_{1}-i+j+1}\left(\frac{2}{3}\right)^{p_{2}+i-j} \zeta_{1}^{i+j} \zeta_{2}^{p_{1}+p_{2}-i-j+1} \mathscr{F}^{i+j, p_{1}+p_{2}-i-j+1}\left(\zeta_{1}, \zeta_{2}\right),
$$

for $0 \leq i \leq p_{1}$ and $0 \leq j \leq p_{2}$. Writing $i+1=i^{\prime}$ in the first of these expressions (and then dropping the ${ }^{\prime}$ ), combining it with the second expression when $1 \leq i \leq p_{1}$ and $0 \leq j \leq p_{2}$, and using the fact that $C_{i-1}^{p_{1}}+C_{i}^{p_{1}}=C_{i}^{p_{1}+1}$, we obtain (16) and (17), with $p_{1}$ replaced by $p_{1}+1$. The outcome is similar if we differentiate partially with respect to $z_{2}$, and (16) and (17) follow by induction.

Now, if $\left(\left|\zeta_{1}\right|,\left|\zeta_{2}\right|\right)$ is normal for $\mathscr{F}$, then

$$
\zeta_{1}^{i+j} \zeta_{2}^{p_{1}+p_{2}-i-j} \mathscr{F}^{i+j, p_{1}+p_{2}-i-j}\left(\zeta_{1}, \zeta_{2}\right)=(1+\sigma) \mathscr{N}_{1}^{i+j} \mathscr{N}_{2}^{p_{1}+p_{2}-i-j} \mathscr{F}\left(\zeta_{1}, \zeta_{2}\right),
$$

from (11), and thus (16) becomes

$$
\mathscr{F}\left(\zeta_{1}, \zeta_{2}\right) \sum_{i=0}^{p_{1}} \sum_{j=0}^{p_{2}} C_{i}^{p_{1}} C_{j}^{p_{2}}\left(\frac{2}{3}\right)^{p_{2}+i-j}\left(-\frac{1}{3}\right)^{p_{1}-i+j}(1+\sigma) \mathscr{N}_{1}^{i+j} \mathscr{N}_{2}^{p_{1}+p_{2}-i-j} .
$$

Rearranging the sum, and using (12) and (7), we obtain

$$
\begin{aligned}
& \sum_{i=0}^{p_{1}} C_{i}^{p_{1}}\left(\frac{2 \mathscr{N}_{1}}{3}\right)^{i}\left(-\frac{\mathscr{N}_{2}}{3}\right)^{p_{1}-i} \sum_{j=0}^{p_{2}} C_{j}^{p_{2}}\left(-\frac{\mathscr{N}_{1}}{3}\right)^{j}\left(\frac{2 \mathscr{N}_{2}}{3}\right)^{p_{2}-j} \\
& \quad+O\left(\mathscr{N}^{* p_{1}+p_{2}-1 / 2}\left(\log \mathscr{N}^{*}\right)^{3 / 2}\right) \\
& =\left(\frac{2 \mathscr{N}_{1}}{3}-\frac{\mathscr{N}_{2}}{3}\right)^{p_{1}}\left(\frac{2 \mathscr{N}_{2}}{3}-\frac{\mathscr{N}_{1}}{3}\right)^{p_{2}}+O\left(\mathscr{N}^{* p_{1}+p_{2}-1 / 2}\left(\log \mathscr{N}^{*}\right)^{3 / 2}\right) \\
& =N_{1}^{p_{1}} N_{2}^{p_{2}}+O\left(N^{* p_{1}+p_{2}-1 / 2}\left(\log N^{*}\right)^{3 / 2}\right) .
\end{aligned}
$$

This proves the theorem, since, from (11) and (7), all other terms on the right hand side of (15) have order at most $N^{* p_{1}+p_{2}-1}$.

\section{Applications to PDEs}

To elucidate our method, we consider some specific examples. It will be useful in what follows to have the following simple cases of (15) to hand:

$$
\begin{aligned}
& 3 z_{1} f^{1,0}\left(z_{1}, z_{2}\right)=2 \zeta_{1} \mathscr{F}^{1,0}\left(\zeta_{1}, \zeta_{2}\right)-\zeta_{2} \mathscr{F}^{0,1}\left(\zeta_{1}, \zeta_{2}\right), \\
& 3 z_{2} f^{0,1}\left(z_{1}, z_{2}\right)=2 \zeta_{2} \mathscr{F}^{0,1}\left(\zeta_{1}, \zeta_{2}\right),-\zeta_{1} \mathscr{F}^{1,0}\left(\zeta_{1}, \zeta_{2}\right) .
\end{aligned}
$$

The order of an entire function $f$ is

$$
\rho(f)=\limsup _{r \rightarrow \infty} \frac{\log \log M(r, r)}{\log r},
$$

so that $\rho(\mathscr{F})=3 \rho(f)$.

Example 3.1. Consider the differential equation

$$
f^{1,0}+f^{0,1}=2 f .
$$


We will show that $\rho(f) \geq 1$. (We remark that this can be done directly since the general solution to $(21)$ is $\Phi\left(z_{1},-z_{2}\right) e^{z_{1}+z_{2}}$, where $\Phi$ is any one dimensional entire function.)

If $\left(\left|\zeta_{1}\right|,\left|\zeta_{2}\right|\right)$ is normal for $\mathscr{F}$ and $\left|\mathscr{F}\left(\zeta_{1}, \zeta_{2}\right)\right|=\mathscr{M}\left(\left|\zeta_{1}\right|,\left|\zeta_{2}\right|\right)$, then by (21), (19), (20) and (11),

$$
\frac{2 \mathscr{N}_{1}-(1+o(1)) \mathscr{N}_{2}}{3 \zeta_{1}^{2} \zeta_{2}}+\frac{2 \mathscr{N}_{2}-(1+o(1)) \mathscr{N}_{1}}{3 \zeta_{1} \zeta_{2}^{2}}=2+o(1)
$$

It follows that

$$
\mathscr{N}^{*} \geq(2+o(1)) \min \left\{\left|\zeta_{1}\right|^{2}\left|\zeta_{2}\right|,\left|\zeta_{1}\right|\left|\zeta_{2}\right|^{2}\right\}
$$

By equation (4.7) in [1] we have

$$
\mathscr{N}_{j} \leq \log \mathscr{M}(\log \log \mathscr{M})^{2}, \quad j=1,2,
$$

and thus

$$
\log \mathscr{M}(\log \log \mathscr{M})^{2} \geq(2+o(1)) \min \left\{\left|\zeta_{1}\right|^{2}\left|\zeta_{2}\right|,\left|\zeta_{1}\right|\left|\zeta_{2}\right|^{2}\right\}
$$

Now the set

$$
T_{K}(R)=\left\{\left(r_{1}, r_{2}\right): 1 \leq \sqrt{r_{1}^{2}+r_{2}^{2}} \leq R \text { and } K^{-1} \leq r_{1} / r_{2} \leq K\right\}
$$

has $\operatorname{logarithmic}$ measure $2 \log K \log R$. Thus, in view of (8), there are arbitrarily large normal values $\left(r_{1}, r_{2}\right)$ for which $K^{-1} \leq r_{1} / r_{2} \leq K$ if $K>e^{3 / 2}$. From this and (23), then, $\rho(\mathscr{F}) \geq 3$, and hence $\rho(f) \geq 1$.

Example 3.2. Consider the $n$-th order linear PDE

$$
f^{n, 0}=\sum_{j=0}^{n} \sum_{i=0}^{n-1} P_{i, j} f^{i, j}
$$

where the $P_{i, j}$ are polynomials in two complex variables. A simple application of the Cauchy-Kovalevskaya Theorem [7] shows that all solutions of (24) are entire and, as in Example 3.1, every solution is transcendental.

To the best of our knowledge there have been no order estimates of entire solutions of (24). Our method can often obtain such results. To simplify matters, let us take the second order equation

$$
f^{2,0}=P f,
$$

where $P$ is a polynomial, and proceed as in Example 3.1. Using (15), (25) becomes

$$
A \zeta_{1} \mathscr{F}^{1,0}+B \zeta_{2} \mathscr{F}^{0,1}+C \zeta_{1}^{2} \mathscr{F}^{2,0}+D \zeta_{1} \zeta_{2} \mathscr{F}^{1,1}+E \zeta_{2}^{2} \mathscr{F}^{0,2}=\zeta_{1}^{4} \zeta_{2}^{2} P\left(\zeta_{1}^{2} \zeta_{2}, \zeta_{1} \zeta_{2}^{2}\right) \mathscr{F},
$$

where $A, B, C, D$ and $E$ are constants. Using (11), we obtain

$$
A \mathscr{N}_{1}+B \mathscr{N}_{2}+C \mathscr{N}_{1}^{2}+D \mathscr{N}_{1} \mathscr{N}_{2}+E \mathscr{N}_{2}^{2}=(1+o(1)) \zeta_{1}^{4} \zeta_{2}^{2} P\left(\zeta_{1}^{2} \zeta_{2}, \zeta_{1} \zeta_{2}^{2}\right),
$$

and therefore

$$
\mathscr{N}^{* 2} \geq(c+o(1))\left|\zeta_{1}\right|^{4}\left|\zeta_{2}\right|^{2}\left|P\left(\zeta_{1}^{2} \zeta_{2}, \zeta_{1} \zeta_{2}^{2}\right)\right|,
$$

where $c$ is a positive constant. As in Example 3.1, this implies that $\rho(\mathscr{F}) \geq 3+3 d$, where $d$ is the degree of $P$, and thus $\rho(f) \geq 1+d$. 
As we have mentioned before, the primary reason to define $\mathscr{F}$ and transform the equation (24) is to be able to apply Theorem 1 with $\sigma \rightarrow 0$ as $\left(r_{1}, r_{2}\right) \rightarrow \infty$ and obtain asyptotically, as in (26), an equation of the form

$$
\mathscr{P}\left(N_{1}, N_{2}\right)=0,
$$

where $\mathscr{P}$ is a polynomial in $N_{1}$ and $N_{2}$ with polynomial coefficients. This parallels the situation in one variable where $(24)$ is an ordinary differential equation and $\mathscr{P}(N)$ is a polynomial in the central index $N$. In such a situation, the possible orders of growth of $N$ can be obtained by inspection or more generally by appealing to the Newton-Puiseux diagram (see e.g. [3]) where it is found that these orders depend only on the degrees of the polynomial coefficients of $\mathscr{P}(N)$.

In many situations the equation (28) allows us to find a minimum growth for $\max \left(\mathscr{N}_{1}, \mathscr{N}_{2}\right)$ and hence a minimum order for a solution to $(25)$. In general, however, there may be significant cancelation among terms of like degree in (28) and, in the extreme, this equation may give us no information at all. Indeed, suppose we take $n=2$ in (25) and transform the equation as before using $\mathscr{F}$. Then provided the degree of the polynomial $Q$ is at least $6, \mathscr{P}$ could well have the form

$$
\mathscr{P}\left(\mathscr{N}_{1}, \mathscr{N}_{2}\right)=\mathscr{N}_{1}^{2}-\frac{1}{2} \mathscr{N}_{2}^{2}-\frac{1}{2} \mathscr{N}_{1} \mathscr{N}_{2}+\mathscr{N}_{1} Q-\mathscr{N}_{2}(Q-3 / 2)+Q-1 .
$$

Except for the fact that the form of $\mathscr{F}$ forces $1 / 2 \leq \mathscr{N}_{1} / \mathscr{N}_{2} \leq 2$, we have no prior knowledge of the relationship between $\mathscr{N}_{1}$ and $\mathscr{N}_{2}$. Conceding the possibility that $\mathscr{N}_{2}=\mathscr{N}_{1}+1$, we find that (29) is identically 0 regardless of the growth of $\max \left(\mathscr{N}_{1}, \mathscr{N}_{2}\right)$.

Example 3.3 One dimensional Wiman-Valiron theory has been successful in showing that certain nonlinear equations cannot have entire solutions. We offer a two dimensional example. Let $P\left(z_{1}, z_{2}\right)$ be a polynomial and consider the equation

$$
f^{1,0} f^{0,1}=P f^{n}, \quad n \geq 3 .
$$

It is easy to check that there is no polynomial solution. We assume that this equation has a transcendental entire solution and proceed as in Example 3.1 to obtain

$$
\frac{2 \mathscr{N}_{1}-(1+o(1)) \mathscr{N}_{2}}{3 \zeta_{1}^{2} \zeta_{2}} \frac{2 \mathscr{N}_{2}-(1+o(1)) \mathscr{N}_{1}}{3 \zeta_{1} \zeta_{2}{ }^{2}}=(1+o(1)) \mathscr{M}\left(\zeta_{1}, \zeta_{2}\right)^{n-2} .
$$

This clearly contradicts (22) proving that (30) has no entire solution.

When $n=2$, (30) may have entire solutions. Indeed $f\left(z_{1}, z_{2}\right)=e^{z_{1} z_{2}}$ is a solution with $P\left(z_{1}, z_{2}\right)=z_{1} z_{2}$. Determining which choices of $P$ allow entire solutions is beyond the scope of our method.

Example 3.4. Consider the differential equation

$$
f^{1,0}=C f^{m} f^{0,1}
$$

where $C$ is a non-zero constant and $m$ is a positive integer. It is easily checked that there are no entire solutions of (31) that are polynomial in one or the other variable, and $\mathrm{Li}[5]$ showed that there are no transcendental entire solutions. Li's proof depends on characterizing common right factors of partial derivatives. We will prove the result using (11) and the following lemma, the proof of which we defer for a moment. 
Lemma 2. There are arbitrarily large positive values of $\lambda$ such that the set

$$
S_{\lambda}:=\left\{r_{2}: r_{2} \geq 1 \text { and }\left(\sqrt{\lambda / r_{2}}, r_{2}\right) \text { is normal }\right\}
$$

has infinite logarithmic measure.

Assume that $f$ is a transcendental solution to (31). We apply the chain rule to $\mathscr{F}$ and use (11) as in the previous examples, evaluating (31) at

$$
Z_{1}=\mathscr{Z}_{1}^{2} \mathscr{Z}_{2}, \quad Z_{2}=\mathscr{Z}_{1} \mathscr{Z}_{2}^{2},
$$

where $\left(\mathscr{Z}_{1}, \mathscr{Z}_{2}\right)$ is such that

$$
\left|\mathscr{F}\left(\mathscr{Z}_{1}, \mathscr{Z}_{2}\right)\right|=\mathscr{M}\left(\left|\mathscr{Z}_{1}\right|,\left|\mathscr{Z}_{2}\right|\right)
$$

and $\left(\left|\mathscr{Z}_{1}\right|,\left|\mathscr{Z}_{2}\right|\right)$ is normal for $\mathscr{F}$. In view of Lemma 2 and the fact that $\left|\mathscr{Z}_{1}\right|=$ $\sqrt{\left|Z_{1}\right| /\left|\mathscr{Z}_{2}\right|}$, we can choose values of $\left|Z_{1}\right|$ (in fact arbitrarily large values, although we do not use that here) such that $\left(\left|\mathscr{Z}_{1}\right|,\left|\mathscr{Z}_{2}\right|\right)$ is normal for arbitrarily large values of $\left|\mathscr{Z}_{2}\right|$. Let us choose and fix such a value of $\left|Z_{1}\right|$.

We first refine the choice of $\mathscr{Z}_{1}$ and $\mathscr{Z}_{2}$. It follows from a result of Hayman [6] that for given $Z_{1}$ and with $f_{Z_{1}}(z):=f\left(Z_{1}, z\right)$, it is possible to choose $Z_{2,0}$ such that $\left|Z_{2,0}\right|=\left|Z_{2}\right|,\left|f_{Z_{1}}\left(Z_{2,0}\right)\right|=\left|f_{Z_{1}}\left(Z_{2}\right)\right|$ and

$$
\frac{Z_{2,0} f_{Z_{1}}^{\prime}\left(Z_{2,0}\right)}{f_{Z_{1}}\left(Z_{2,0}\right)} \geq \frac{\left|Z_{2,0}\right| M^{\prime+}\left(\left|Z_{2,0}\right|, f_{Z_{1}}\right)}{M\left(\left|Z_{2,0}\right|, f_{Z_{1}}\right)},
$$

the left hand side being real. Here ${ }^{\prime+}$ represents the right hand derivative. We replace our original $Z_{2}$ by $Z_{2,0}$, and solve (33) for $\mathscr{Z}_{1}$ and $\mathscr{Z}_{2}$. The upshot is that we may assume that (35) holds at $Z_{2}$.

From (31), (19) and (20), we obtain

$$
\left(2 \mathscr{N}_{1}-(1+o(1)) \mathscr{N}_{2}\right)=(C+o(1))\left(2 \mathscr{N}_{2}-\left(1+o(1) \mathscr{N}_{1}\right)\left(\mathscr{Z}_{1} / \mathscr{Z}_{2}\right) \mathscr{F}^{m},\right.
$$

and from this and $(22)$ it follows that $2 \mathscr{N}_{2}=(1+o(1)) \mathscr{N}_{1}$. Returning to (31), and now using (11) to rewrite only the left hand side, we have

$$
\begin{aligned}
\left(3 C^{-1}+o(1)\right) \frac{Z_{2} \mathscr{N}_{2}}{\mathscr{Z}_{1} \mathscr{F}^{m}\left(\mathscr{Z}_{1}, \mathscr{Z}_{2}\right)} & =\frac{Z_{2} f^{0,1}\left(Z_{1}, Z_{2}\right)}{f\left(Z_{1}, Z_{2}\right)}=\frac{Z_{2} f_{Z_{1}}^{\prime}\left(Z_{2}\right)}{f_{Z_{1}}\left(Z_{2}\right)} \\
& \geq \frac{\left|Z_{2}\right| M^{\prime+}\left(\left|Z_{2}\right|, f_{Z_{1}}\right)}{M\left(\left|Z_{2}\right|, f_{Z_{1}}\right)} .
\end{aligned}
$$

Since $M\left(\left|Z_{2}\right|, f_{Z_{1}}\right)=M\left(\left|Z_{1}\right|,\left|Z_{2}\right|\right)$ and $M\left(1, f_{Z_{1}}\right) \leq M\left(\left|Z_{1}\right|, 1\right)$, we have

$$
\begin{aligned}
\frac{\left|Z_{2}\right| M^{\prime}\left(\left|Z_{2}\right|, f_{Z_{1}}\right)}{M\left(\left|Z_{2}\right|, f_{Z_{1}}\right)} & \geq \frac{\log M\left(\left|Z_{2}\right|, f_{Z_{1}}\right)-\log M\left(1, f_{Z_{1}}\right)}{\log \left|Z_{2}\right|} \\
& \geq \frac{\log M\left(\left|Z_{1}\right|,\left|Z_{2}\right|\right)-\log M\left(\left|Z_{1}\right|, 1\right)}{\log \left|Z_{2}\right|} .
\end{aligned}
$$

As we have observed, the left hand side of $(37)$ tends to zero as $\left(\mathscr{Z}_{1}, \mathscr{Z}_{2}\right) \rightarrow \infty$. On the other hand, since $\left|Z_{1}\right|$ is fixed, the right hand side of (38) tends to $\infty$, a contradiction.

Turning to the proof of Lemma 2, let us recall the way in which the normal set in two dimensional Wiman-Valiron theory arises. The method of [1] defines a tiling of the $\left(\log r_{1}, \log r_{2}\right)$ plane by a collection of non-overlapping, convex polygons. 
Each polygon is assigned, in a certain way, an ordered pair of non-negative integers $\left(N_{1}, N_{2}\right)$. A sequence of numbers, $\rho_{N}(N=0,1,2 \ldots)$, is given, satisfying

$$
1 \leq \rho_{N} \leq e^{2} \quad \text { and } \quad \rho_{N} \rightarrow e^{2} \text { as } N \rightarrow \infty,
$$

and the polygon to which $\left(N_{1}, N_{2}\right)$ is assigned is translated by the vector $\left(\log \rho_{N_{1}}, \log \right.$ $\rho_{N_{2}}$ ). The translated polygons are non-overlapping, but the translation introduces gaps between them. The normal points $\left(r_{1}, r_{2}\right)$ are those for which $\left(\log r_{1}, \log r_{2}\right)$ lies in the interior of the translated polygons. Also, if $\left(r_{1}, r_{2}\right)$ is normal, the pair $\left(N_{1}, N_{2}\right)$ assigned to the polygon to which $\left(\log r_{1}, \log r_{2}\right)$ belongs is the central index at $\left(r_{1}, r_{2}\right)$.

To prove Lemma 2, consider, for $m, n \in \mathbf{N}$,

$$
\mathscr{P}_{m, n}=\left\{\left(\sqrt{\lambda / r_{2}}, r_{2}\right): \lambda \in\left[e^{8 m}, e^{8 m+8}\right] \text { and } r_{2} \in\left[e^{8 n}, e^{8 n+8}\right]\right\} \text {. }
$$

This set corresponds to a parallelogram $\mathscr{Q}_{m, n}$ in the $\left(\log r_{1}, \log r_{2}\right)$ plane, with vertices $(4 m-4 n, 8 n),(4 m-4 n+4,8 n),(4 m-4 n-4,8 n+8)$ and $(4 m-4 n, 8 n+$ 8). From the preceding remarks, the part of $\mathscr{Q}_{m, n}$ that corresponds to exceptional points is no larger than the set of points that are translated out of $\mathscr{Q}_{m, n}$ by the vector $\left(\log \rho_{N_{1}}, \log \rho_{N_{2}}\right)$. Both components of $\left(\log \rho_{N_{1}}, \log \rho_{N_{2}}\right)$ are positive and $\left|\left(\log \rho_{N_{1}}, \log \rho_{N_{2}}\right)\right| \leq 2 \sqrt{2}$, from (39). Also, the shortest distance from the bottom left hand corner of $\mathscr{Q}_{m, n}$ to the right hand sloping side is $8 / \sqrt{5}>2 \sqrt{2}$. There is thus a small parallelogram $\mathscr{R}_{m, n}$ in the bottom left hand corner of $\mathscr{Q}_{m, n}$, similar to $\mathscr{Q}_{m, n}$ and having the same dimensions for all $m$ and $n$, that is not translated outside $\mathscr{Q}_{m, n}$. If the area of $\mathscr{R}_{m, n}$ is $C$ say, then the logarithmic measure of the normal set in $\mathscr{P}_{m, n}$ is at least $C$. Thus, making the change of variables $\left(r_{1}, r_{2}\right) \rightarrow\left(\lambda, r_{2}\right)$, where $\lambda=r_{1}^{2} r_{2}$, so that

and defining

$$
\frac{d \lambda d r_{2}}{\lambda r_{2}}=2 \frac{d r_{1} d r_{2}}{r_{1} r_{2}}
$$

$$
S_{\lambda, n}=\left\{r_{2}: e^{8 n+8} \geq r_{2} \geq e^{8 n} \text { and }\left(\sqrt{\lambda / r_{2}}, r_{2}\right) \text { is normal }\right\},
$$

and also

we have

$$
I_{n}(\lambda)=\int_{S_{\lambda, n}} \frac{d r_{2}}{r_{2}}
$$

$$
\int_{e^{8 m}}^{e^{8 m+8}} \frac{I_{n}(\lambda)}{\lambda} d \lambda \geq 2 C .
$$

On the other hand, if the set (32) has finite logarithmic measure for all $\lambda \in\left[e^{8 m}, e^{8 m+8}\right]$, then $I_{n}(\lambda) \rightarrow 0$ as $n \rightarrow \infty$ for every $\lambda \in\left[e^{8 m}, e^{8 m+8}\right]$, and therefore the integral on the left hand side of (40) tends to 0 as $n \rightarrow \infty$, a contradiction.

\section{References}

[1] Fenton, P. C.: Wiman-Valiron theory in two variables. - Trans. Amer. Math. Soc. 347, 1995, 4403-4412.

[2] Fenton, P. C.: Polynomial approximation of entire functions of two variables. - Complex Var. Theory Appl. 40, 2000, 211-230.

[3] Jank, G., and L. Volkmann: Einführung in die Theorie der ganzen und mermomorphen Funktionen mit Anwendungen auf Differentialgleichungen. - Birkhäuser, Basel-Boston, 1985. 
[4] Laine, I.: Nevanlinna theory and complex differential equations. - de Gruyter, Berlin, 1993.

[5] LI, B. Q.: Entire solutions of eiconal type equations. - Arch. Math. (Basel) 89, 2007, 350-357.

[6] Hayman, W. K.: A characterization of the maximum modulus of functions regular at the origin. - J. Analyse Math. 1:1, 1951, 135-154.

[7] Renardy, M., and R. Rogers: An introduction to partial differential equations. - Springer, Heidelberg, 1993.

Received 19 October 2009 